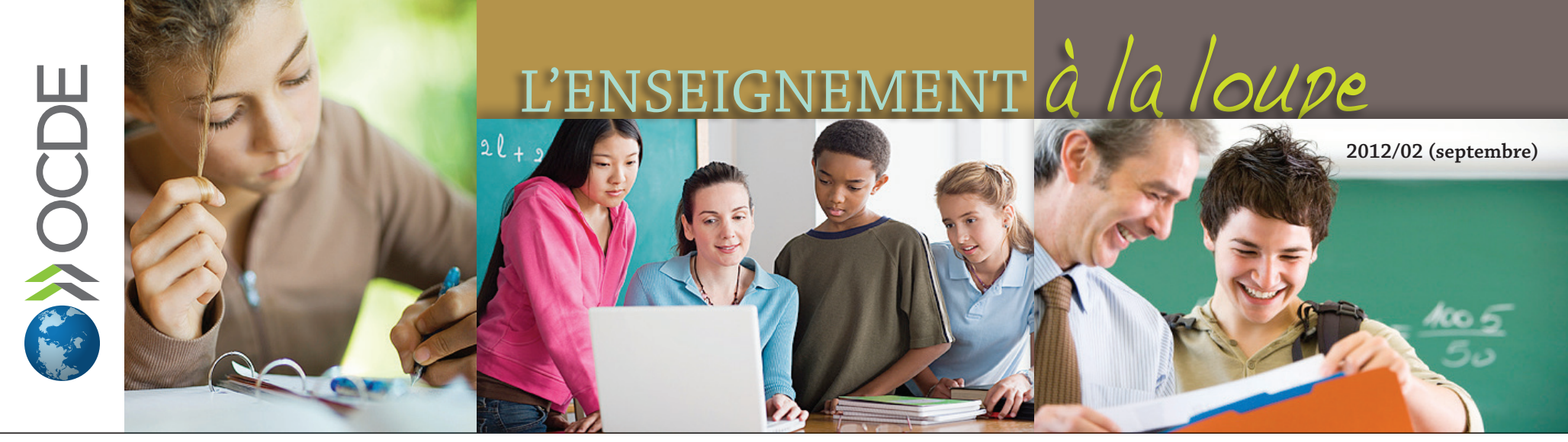

\title{
Enseignants débutants : quel soutien leur apporter ?
}

- Les établissements soutiennent les enseignants débutants en leur proposant des programmes de tutorat et d'initiation, mais près d'un tiers des enseignants débutants font part de besoins importants de formation continue en matière de discipline en classe et de problèmes de comportement des élèves.

- Contrairement aux idées reçues, les établissements dans lesquels travaillent les enseignants débutants ne sont pas différents de ceux où exercent leurs collègues plus expérimentés.

- Selon les pays participant à l'Enquête internationale sur l'enseignement et l'apprentissage (TALIS), les enseignants débutants ${ }^{1}$ consacrent moins de temps à l'enseignement et l'apprentissage, et davantage de temps à la gestion de leur classe que leurs collègues plus expérimentés ; ils font en outre part de niveaux inférieurs de sentiment d'efficacité personnelle.

\section{Nombreux sont ceux qui se lancent dans la profession d'enseignant, mais n'y restent pas...}

Lorsqu'ils débutent dans la profession, les enseignants se retrouvent face à des classes d'élèves à la fois passionnantes et remplies de défis. Cependant, en moyenne, près de $10 \%$ des enseignants quittent définitivement la profession au cours de leurs trois premières années d'enseignement, avec parfois un taux de départs supérieur de $150 \%$ à celui des enseignants plus expérimentés (OCDE, 2005). Afin de faciliter l'épanouissement et la réussite professionnels des enseignants débutants, il est essentiel de comprendre dans quels domaines leurs besoins sont les plus importants et comment les aider à maîtriser pleinement leurs compétences professionnelles. L'offre de ce type de soutien peut aider à maintenir les enseignants débutants dans la profession.

$$
\text { TALIS, gu'est-ce gue c'est? }
$$

TALIS est la première enquête internationale s'intéressant aux environnements d'enseignement et d'apprentissage dans les établissements d'enseignement. L'enquête TALIS 2008 a interrogé des enseignants et des chefs d'établissement de 24 pays sur leur travail, leur établissement et leur(s) classe(s). (L'édition 2013 de l'enquête est actuellement administrée dans 33 pays.) Grâce à son analyse comparative internationale, elle aide les pays à identifier d'autres pays confrontés à des enjeux similaires, leur permettant ainsi de s'inspirer de leurs politiques respectives.

Les données de cette synthèse sont principalement tirées du rapport The Experience of New Teachers: Results from TALIS 2008. Pour tout complément d'information, consulter www.oecd.org/talis.

\section{Les enseignants débutants ont moins confiance en leur efficacité...}

Les pays s'intéressent à l'efficacité de leurs enseignants, qu'ils soient débutants ou plus expérimentés, et nombre d'entre eux également à la façon dont les établissements de formation initiale des enseignants les préparent à l'entrée dans la profession. L'enquête TALIS n'évalue pas l'efficacité des enseignants, mais les interroge sur leur propre sentiment d'efficacité personnelle. En moyenne, les enseignants débutants font ainsi part d'un sentiment d'efficacité personnelle significativement inférieur à celui de leurs collègues plus expérimentés (voir le graphique 1).

1. Par enseignants débutants, on entend ceux ayant deux années ou moins d’expérience rémunérée dans la profession. 


\section{Graphique 1 • Sentiment d'efficacité personnelle chez les enseignants débutants et leurs collègues plus expérimentés}

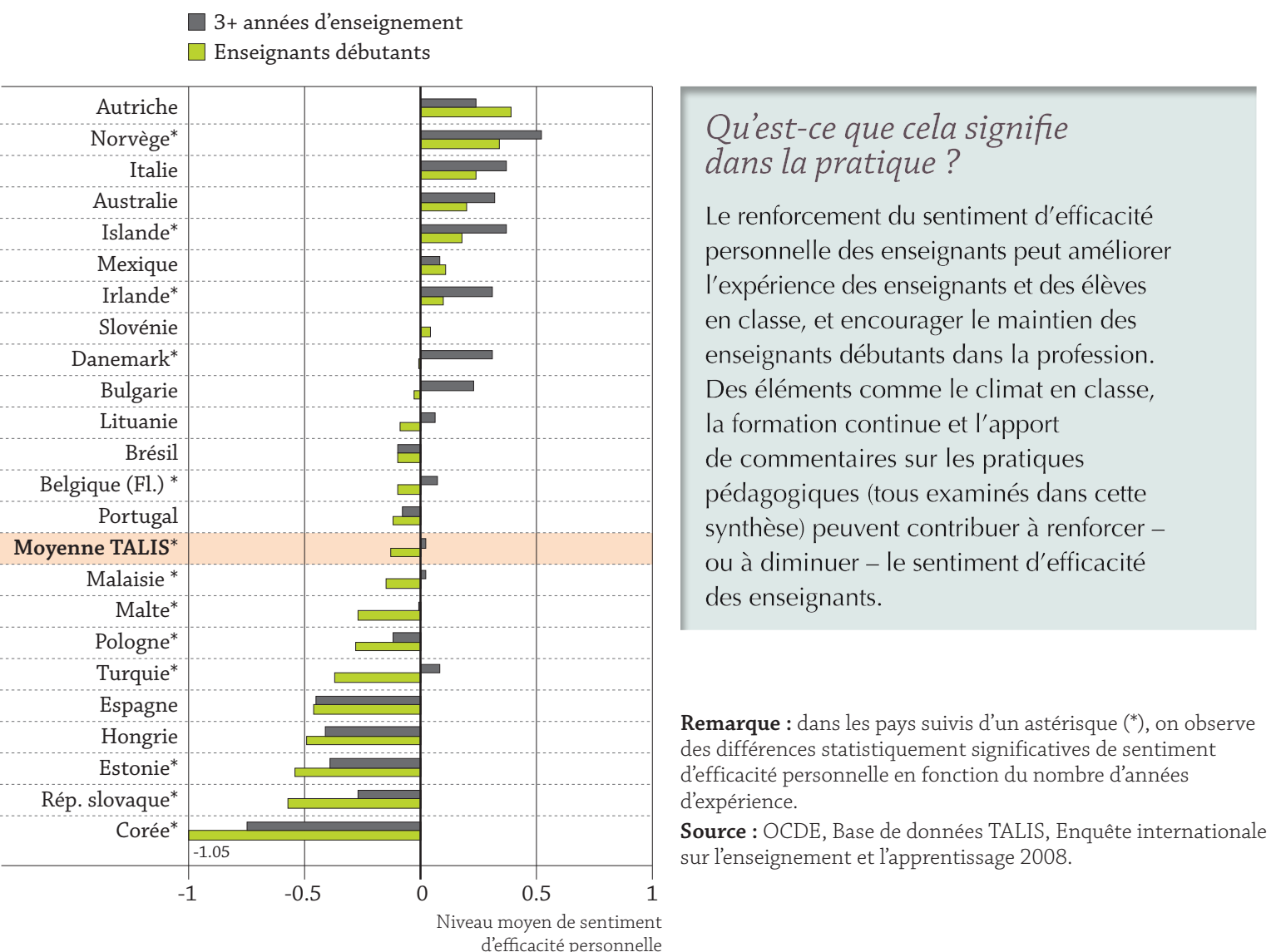

\section{Les conditions de travail des enseignants débutants sont similaires à celles de leurs collègues plus expérimentés...}

Dans les pays où les enseignants débutants quittent la profession en grand nombre, leur taux de départs est souvent mis sur le compte de leur nomination dans des établissements où les conditions de travail sont plus dures ou les postes plus difficiles à pourvoir.

Selon les données de l'enquête TALIS, les classes des enseignants débutants sont en moyenne similaires à celles de leurs collègues plus expérimentés. La situation linguistique ou le milieu socio-économique de leurs élèves ne diffère quasiment pas, et leurs établissements présentent des niveaux similaires de ressources matérielles et humaines. Dans sept des pays de l'enquête TALIS, les enseignants débutants bénéficient d'une charge d'enseignement moins lourde que celle de leurs collègues plus expérimentés. Le seul pays où l'inverse s'observe est l'Australie. En outre, la moitié des pays participant à l'enquête TALIS contribuent à faciliter la réussite des enseignants débutants en leur attribuant des classes dont les effectifs sont significativement réduits.

\section{Qu'est-ce que cela signifie dans la pratique?}

Bien que les conditions de travail des enseignants débutants ne soient pas nécessairement plus difficiles que celles de leurs collègues plus expérimentés, ils ont toutefois moins confiance en leur capacité à enseigner. Repenser l'organisation scolaire pour alléger la charge d'enseignement des enseignants débutants pourrait permettre de dégager du temps pour la planification des leçons, l'offre d'un soutien de la part des équipes de l'établissement et l'observation en classe. 


\section{Lorsqu'ils débutent, les enseignants consacrent davantage de temps à la gestion de la classe et moins de temps à l'enseignement...}

Les enseignants débutants indiquent consacrer considérablement plus de temps à la gestion de leur classe que leurs collègues plus expérimentés (voir le graphique 2). En moyenne, les enseignants débutants consacrent ainsi $9 \%$ de leur temps à des tâches administratives, $18 \%$ au maintien de l'ordre et à la gestion de la classe, et $73 \%$ à l'enseignement et à l'apprentissage proprement dits.

\section{Graphique 2 • Différences de temps d'enseignement entre les enseignants débutants} et leurs collègues plus expérimentés, par pays

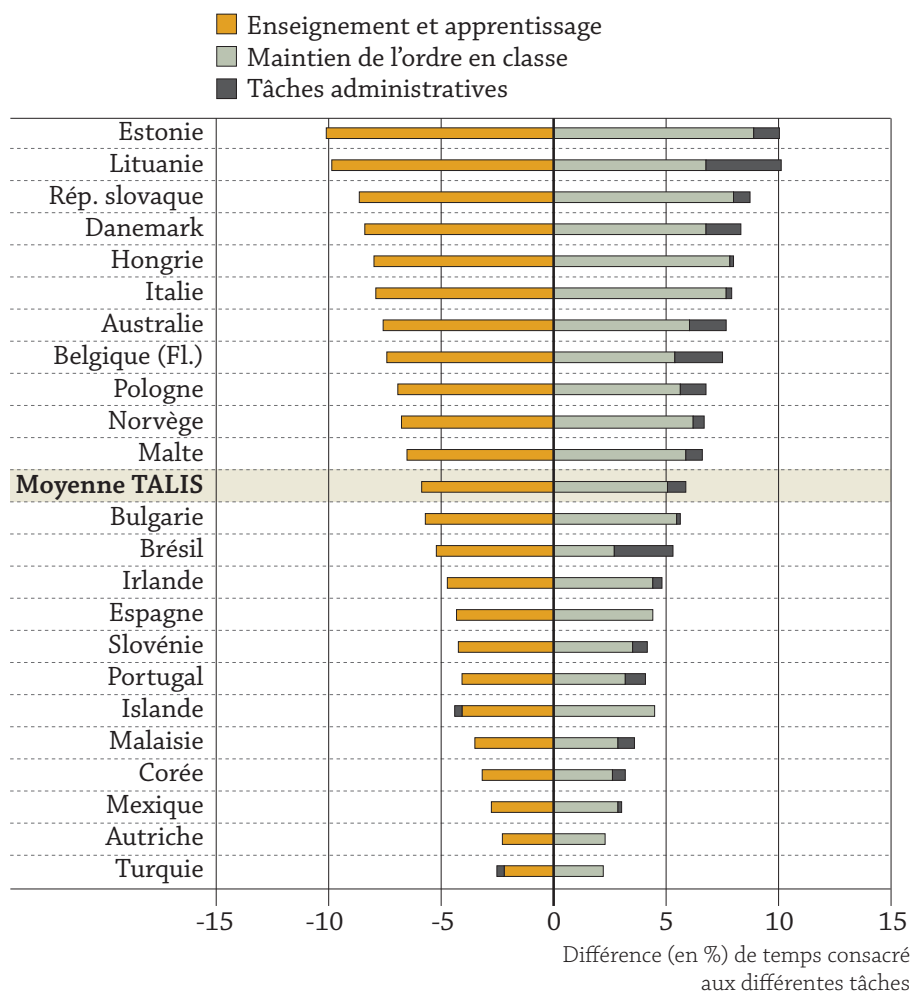

Source : OCDE, Base de données TALIS, Enquête internationale sur l'enseignement et l'apprentissage 2008.

Plus on consacre de temps à la gestion de la classe ou à la discipline, moins on en consacre à l'enseignement durant chaque séance. Un enseignant débutant sur quatre fait part de besoins importants de formation continue en matière de discipline et de problèmes de comportement des élèves, une proportion qui ne s'établit qu'à un sur huit parmi les enseignants plus expérimentés.

\section{Qu'est-ce que cela signifie dans la pratique} Les enseignants débutants sont conscients de leurs besoins en matière de gestion de la classe et soucieux de s'améliorer. La mise en place de dispositifs de formation professionnelle continue au sein même des établissements afin d'améliorer les compétences de gestion de la classe pourrait permettre de répondre à ces besoins et d'accroître le temps consacré à l'enseignement et l'apprentissage.

\section{De nombreux établissements soutiennent les enseignants débutants dès le départ...}

Dans les pays participant à l'enquête TALIS, les établissements mettent en place des programmes de tutorat et d'initiation pour soutenir les enseignants débutants (voir le graphique 3). Trois quarts des enseignants débutants ayant participé à l'enquête travaillent ainsi dans un établissement proposant des programmes de tutorat et/ou d'initiation. La qualité de ces programmes varie toutefois considérablement : alors que certains ne sont que des introductions administratives, d'autres prennent la forme de partenariats pouvant durer plusieurs années. 


\section{Graphique 3 • Pourcentage d'établissements proposant des programmes d'initiation aux enseignants débutants, par pays}

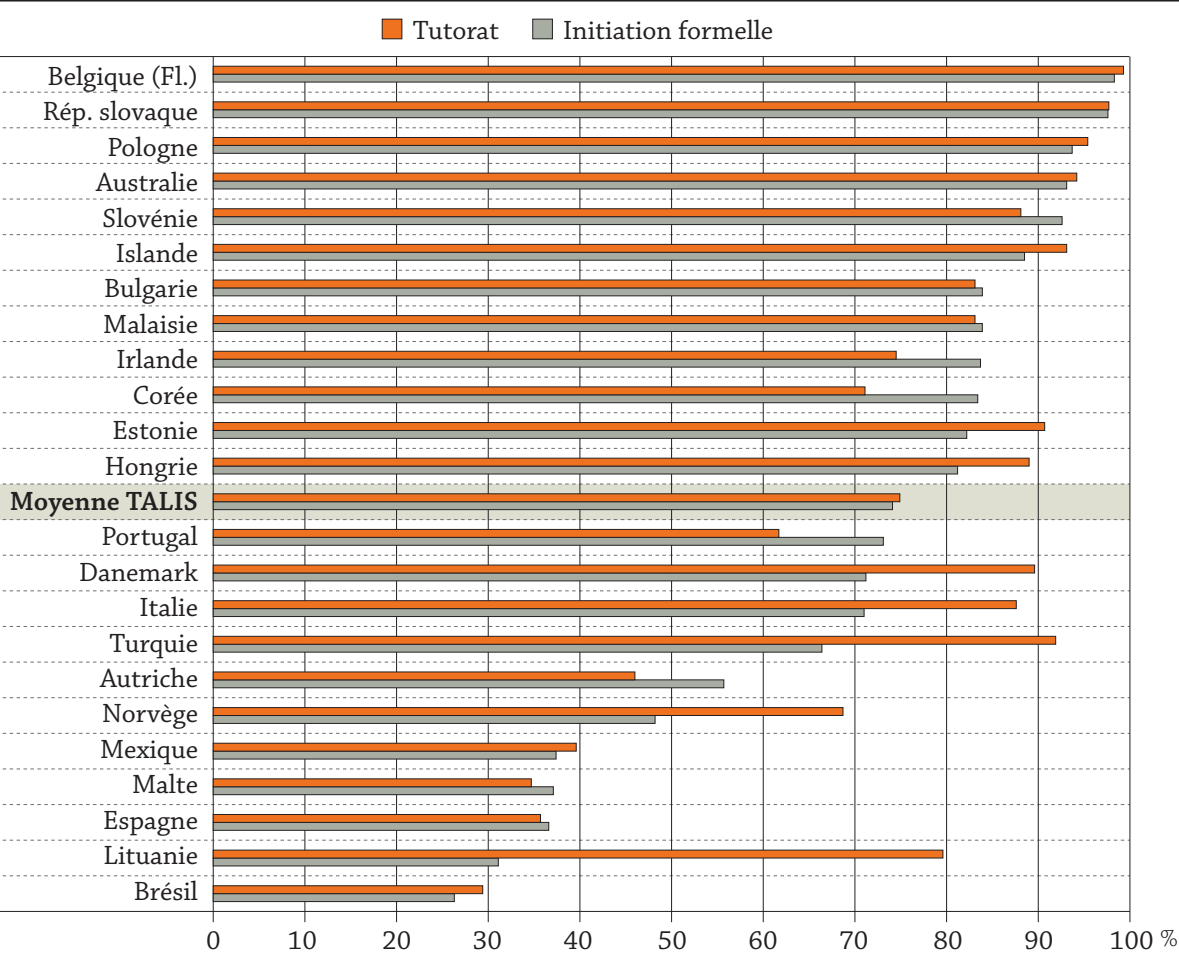

Source : OCDE, Base de données TALIS, Enquête internationale sur l'enseignement et l'apprentissage 2008.

Étonnamment, il semble n'exister aucune relation entre l'offre de ce type de programmes et le fait que les enseignants débutants bénéficient de commentaires sur leur enseignement. Plus de la moitié des enseignants débutants travaillant dans des établissements proposant des programmes d'initiation ou de tutorat indiquent ainsi ne recevoir des commentaires qu'une fois par an, voire moins. Face à des enseignants débutants désireux d'améliorer leurs pratiques pédagogiques, il pourrait s'agir là d'une véritable occasion manquée pour les établissements. L'évaluation des enseignants débutants et les commentaires qui s'ensuivent ne doivent pas nécessairement s'inscrire dans un processus formel, mais peuvent s'avérer des outils précieux de formation continue et de soutien supplémentaire.

Pour conclure Il est vrai que la responsabilité de la préparation des enseignants débutants à l'entrée dans la profession incombe principalement aux établissements de formation initiale. Cependant, une fois les enseignants débutants dans la profession, quelle que soit la situation de leur établissement ou de leur classe, les établissements disposent de différents moyens pour leur apporter davantage de soutien. Lallégement de la charge d'enseignement, l'apport de commentaires dans le cadre de programmes de tutorat et l'offre d'activités de formation continue en matière de gestion de la classe apparaissent ainsi comme autant d'outils facilement accessibles à même d'améliorer le sentiment d'efficacité personnelle des enseignants débutants et d’augmenter leurs chances de succès.

\section{Référence}

OCDE (2005), Le rôle crucial des enseignants : Attirer, former et retenir des enseignants de qualité, Éditions OCDE, Paris, http://dx.doi.org/ $10.1787 / 9789264018051$-fr.

\section{Voir}

www.oecd.org/talis

\section{Contacter}

Kristen Weatherby (Kristen.Weatherby@oecd.org)
Pour en savoir plus

Consulter The Experience of New Teachers: Results from TALIS 2008

Ce document est publié sous la responsabilité du Secrétaire général de l’OCDE. Les opinions qui y sont exprimées et les arguments qui y sont employés ne reflètent pas nécessairement les vues officielles des pays membres de l'OCDE.

Ce document et toute carte qu'il peut comprendre sont sans préjudice du statut de tout territoire, de la souveraineté s'exerçant sur ce dernier, du tracé des frontières et limites internationales, et du nom de tout territoire, ville ou région.

Les données statistiques concernant Israël sont fournies par et sous la responsabilité des autorités israéliennes compétentes. L'utilisation de ces données par l'OCDE est sans préjudice du statut des hauteurs du Golan, de Jérusalem-Est et des colonies de peuplement israéliennes en Cisjordanie aux termes du droit international. 